\title{
Excitation Properties and Cryogenic Stability of Helical Coils for the LHD
}

\author{
S. Imagawa, N. Yanagi, T. Mito, H. Chikaraishi, S. Hamaguchi, H. Sekiguchi, S. Yamada, T. Satow, \\ Y. Nakamura, S. Satoh, and O. Motojima
}

\begin{abstract}
The helical coils for the Large Helical Device are the world's largest pool-cooled superconducting coils in operation. These were expected to be cryostable up to $13.0 \mathrm{kA}$ at 4.4 $\mathrm{K}$ on a basis of the measured recovery currents in all the short samples. However, a normal-zone was induced at higher than $11 \mathrm{kA}$ repeatedly. It propagated to the finite length and recovered within several seconds except at $11.45 \mathrm{kA}$. Because of slow current diffusion into a pure aluminum stabilizer, a normal zone can propagate dynamically below the cold-end recovery current. The excitation tests have been carried out, and average $11.65 \mathrm{kA}$ has been achieved by grading the current in the three blocks of the helical coil. The disturbance during excitations and the cryogenic stability of the coil are presented.
\end{abstract}

Index Terms-aluminum stabilizer, minimum propagating current, dynamic stability, helical coil

\section{INTRODUCTION}

$\mathrm{T}$ HE Large Helical Device (LHD) is the largest stellarator which has been in operation from 1998 to study fusion plasma close to a reactor region [1]. The helical coils are pool-cooled superconducting coils that produce a twisted toroidal magnetic field. The conductor consists of $\mathrm{NbTi}$ strands, a pure aluminum stabilizer and a copper sheath to realize enough cryogenic stability and mechanical strength. The coils were designed to satisfy 'cold-end' stability for the design current of $13 \mathrm{kA}$ by using recovery currents of the short samples that were measured after inducing a certain length of normal zone by a heater [2]. However, dynamic calculations predicted that a normal zone propagates below the cold-end recovery current by excess heat generation during the current diffusing into the thick pure aluminum stabilizer [3-4]. This effect was confirmed in a test coil of 10 turns. The first propagation of a normal zone in the real helical coil was observed in the first excitation up to 11.25 kA [5]. The normal zone expanded to about $2 \mathrm{~m}$ and recovered. A wide propagation over $10 \mathrm{~m}$ occurred in the following excitation up to $11.45 \mathrm{kA}$, and it could not recover finally, because the accumulated helium bubbles would deteriorate the cooling condition. The excitation tests have been carried out in the intervals of plasma experiments to attain the higher magnetic field. This paper intends to investigate the recent results and to discuss the cryogenic stability of the helical coils.

Manuscript received September 17, 2000

$S$. Imagawa and other authors are with the National Institute for Fusion Science, 322-6 Oroshi-cho, Toki, Gifu 509-5292, Japan (telephone: $+81-$ 572-58-2132, e-mail: imagawa@LHD.nifs.ac.jp).

\section{HeLical COIL SYSTEM OF LHD}

The coil system of the LHD consists of a pair of helical coils and three pairs of poloidal coils. In order to change the current center and to reduce the voltage during a current shut-off, the helical coil is divided into three blocks that are called $\mathrm{H}-\mathrm{I}, \mathrm{H}-\mathrm{M}$ and $\mathrm{H}-\mathrm{O}$ from the bottom, as shown in Fig. 1. Each pair of the blocks is usually connected in series, as shown in Fig. 2. Each power supply has an own local control unit, and the necessary voltage is calculated every $20 \mathrm{~ms}$ by the central control unit to minimize the current errors [6]. A simple $P$ control scheme is adopted in the usual operation, and the feedback gain $K_{p}$ is set to 0.1 to prevent hunting. The current error less than $0.2 \%$ is realized for the helical coils, and the time constant of current feedback is about $10 \mathrm{~s}$.

Sensors are not installed on the conductors to avoid troubles due to these cables or feed-throughs. Voltage taps are attached on the flexible joint pieces between the coil leads and the superconducting bus-lines. The coil cases are equipped with 38 temperature sensors, 45 strain gauges and 10 Hall sensors. Temperature sensors are attached on the joint pieces. AE sensors are not installed yet.

The voltages are monitored by a pen recorder and recorded by fast data acquisition devices. A low-pass filter is adopted to distinguish the signals of the coil voltages in the order of $\mathrm{mV}$ from background noises. The cutting frequency is set to $10 \mathrm{~Hz}$, and the fastest sampling rate is $50 \mathrm{~Hz}$. The other slow data are recorded by several data acquisition systems. The coil quench is detected by the balance voltage which is the difference between the pair of the coil blocks.

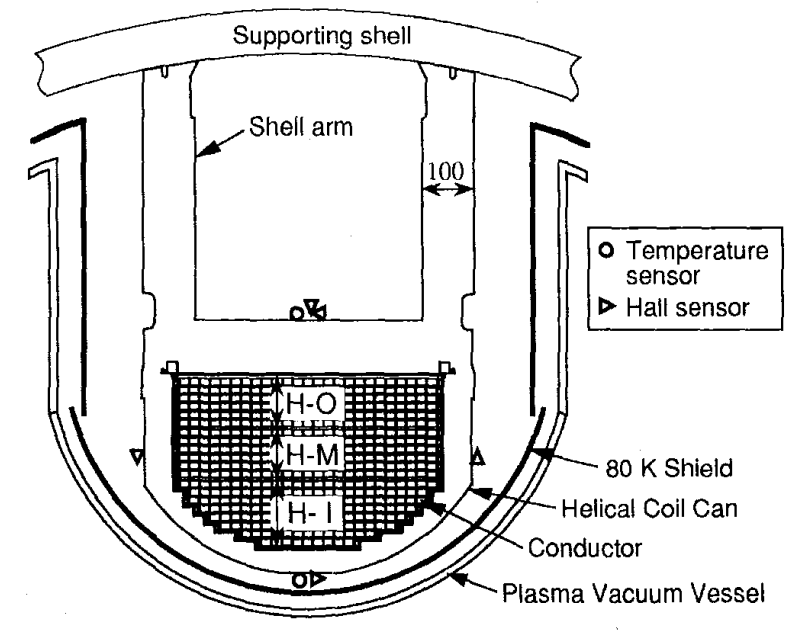

Fig. 1. Cross-sectional view of the helical coil for LHD. 


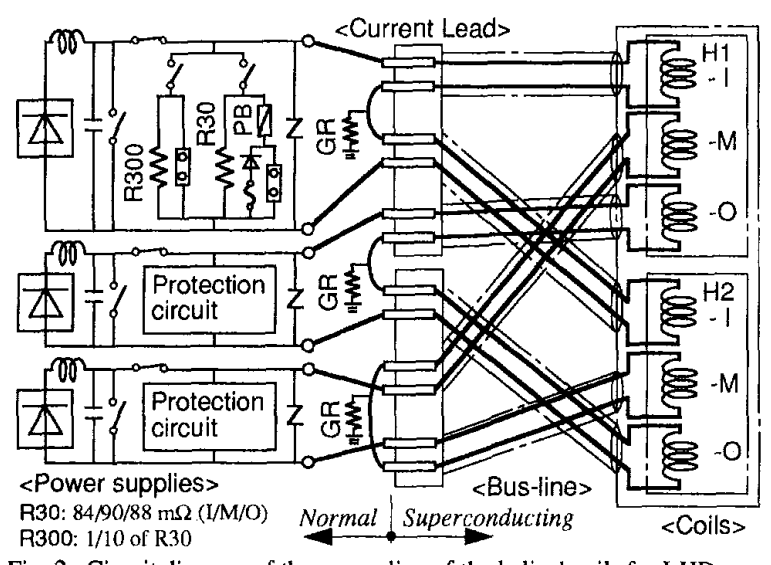

Fig. 2. Circuit diagram of the power line of the helical coils for LHD.

\section{BALANCE VOLTAGE}

\section{A. Balance Voltage by Conductor Motions}

In order to investigate the balance voltages of the coils, we introduce general equations for three strongly coupled circuits of coils. When a motion occurs in the coil 1 while keeping all the currents $I_{i}(i=1$ to 3 ) constant, the induced voltages $e_{i}$ are expressed as

$$
\begin{aligned}
& e_{1}=\dot{L}_{1} I_{1}+\dot{M}_{12} I_{2}+\dot{M}_{13} I_{3}+\varepsilon \\
& e_{i}=\dot{M}_{1 i} I_{1} \quad(i=2,3)
\end{aligned}
$$

where $L$ and $M$ are self and mutual inductances, and $\varepsilon$ is the voltage due to the work load by the motion. On the other hand, the change of the magnetic energy $\dot{W}_{0}$ is expressed as

$$
\dot{W}_{0}=\dot{L}_{1} I_{1}^{2} / 2+\dot{M}_{12} I_{1} I_{2}+\dot{M}_{13} I_{1} I_{3}
$$

When the motion does a mechanical work $W_{f}$, the following equation is derived from the law of energy conservation.

$$
e_{1} I_{1}+e_{2} I_{2}+e_{3} I_{3}=\dot{W}_{0}+\dot{W}_{f}
$$

From (2) to (4), $e_{1}$ is expressed as

$$
e_{1}=\dot{L}_{1} I_{1} / 2+\dot{W}_{f} / I_{1}
$$

When the motion is small, (2) is approximately expressed as

$$
e_{i}=d\left(k_{1 i} \sqrt{L_{1} L_{i}}\right) / d t \cdot I_{1}=k_{1 i} \sqrt{L_{i} / L_{1}} \cdot \dot{L}_{1} I_{1} / 2(i=2,3)
$$

where $k_{1 i}$ is the coupling coefficient between coil 1 and 2 or 3. In the case of $L_{1} \approx L_{2} \approx L_{3}, e_{1}$ is higher than $e_{2}$ or $e_{3}$, because both $\dot{L}_{1}$ and $\dot{W}_{f}$ are positive for a motion induced by electromagnetic forces, and because $k_{1 i}<1$.

\section{B. Balance Voltage by Propagation of a Normal Zone}

When a normal zone propagates, the current diffuses into an aluminum stabilizer. Since the geometrical center of the stabilizer is shifted from the superconducting strands, as shown in Fig. 3, the self inductance becomes smaller. In this case, any mechanical works are not induced, but the voltage drop $V_{R}$ due to the resistance of the normal zone is added.

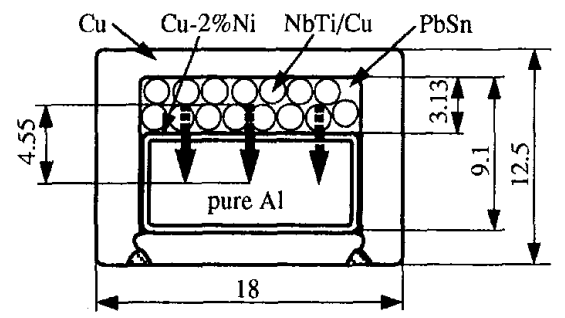

Fig. 3. Cross-section of the helical coil conductor and schematic drawing of current diffusion.

When the propagation occurs in the coil 1 , the voltage is derived from (5) as

$$
e_{1}=\dot{L}_{1} I_{1} / 2+V_{R} \text {. }
$$

From (6) and (7), $V_{R}$ is expressed as

$$
V_{R}=e_{1}-\sqrt{L_{1} / L_{2}} / k_{12} \cdot e_{2} .
$$

Furthermore, the work load by the shift of the current center is expressed as $-B \cdot I_{1} \cdot 2 u \cdot a$, where $B, u, a$ are the magnetic field perpendicular to the direction of the current shift, the propagation velocity, and the length of the shift, respectively. Since this work load is equal to the change of the magnetic energy, the following equation is derived from (3) and (6).

$$
2 u=\frac{-1}{B a}\left(1+k_{12} \sqrt{\frac{L_{2}}{L_{1}}} \frac{I_{2}}{I_{1}}+k_{13} \sqrt{\frac{L_{3}}{L_{1}}} \frac{I_{3}}{I_{1}}\right) \frac{1}{k_{12}} \sqrt{\frac{L_{1}}{L_{2}}} e_{2} .
$$

In the case of LHD, $L_{I}=1.260 \mathrm{H}, L_{M}=1.279 \mathrm{H}, L_{O}=$ $1.343 \mathrm{H}, k_{I M}=0.91$, and $k_{I O}=0.808$ where the subindex corresponds to the name of each block of the helical coil. When a normal zone is induced in the H-I block, the voltage drop by the resistance can be estimated by

$$
V_{R}=e_{l}-e_{M} / 0.917 \text {. }
$$

Besides, the voltage is also estimated by an integration of the propagation velocity given as (9). When $I_{i}=11.45 \mathrm{kA}, B=$ $5.3 \mathrm{~T}$, and the resistance of the conductor $r=0.77 \mu \Omega / \mathrm{m}, V_{R}$ is estimated by

$$
V_{R}=r \cdot I_{l} \int 2 u d t=-1.1 \int e_{M} d t
$$

\section{EXCITATION TEST}

\section{A. Method and Procedure}

The helical coils for LHD are cooled by saturated liquid helium which is supplied from the 10 bottoms to keep the liquid level in a header tank. The gas returns to the suction of compressors through heat exchangers, and the pressure is around $0.115 \mathrm{MPa}$ that corresponds to $4.4 \mathrm{~K}$. All the coils are charged simultaneously by keeping the ratio of currents. Five standard ratios were named \#1-0, a, b, c and d, which correspond to the shapes of magnetic surfaces of quasi-circle, vertical elongation, horizontal elongation, outward shift, and inward shift, respectively.

The excitation tests in the first cooling period were limited 
up to $1.5 \mathrm{~T}$ to confirm the reliability of the whole system. The major excitation tests in the second and third cooling periods are listed in Table 1. After a total of 10 weeks' plasma experiments at $1.5 \mathrm{~T}$, the excitation test up to $3.0 \mathrm{~T}$ of \#1-0 was tried, where the current of the helical coils would reach $12.5 \mathrm{kA}$. In spite of the cryostable design, a normal zone propagated widely when reaching $11.45 \mathrm{kA}$ at a ramp rate of $0.1 \mathrm{~T} / \mathrm{min}$. In the following excitations, the ramp rate was reduced to $1 / 10$ at higher than $10 \mathrm{kA}$ in order to keep good cooling condition. The second excitation up to 11.45 kA was attained without inducing any normal zones. A kind of training effect was observed. The number and amplitude of the spike voltages decreased by repeating excitations [7].

\section{B. Latest Results}

Typical balance voltages in charging and discharging processes are shown in Fig. 4. The spike voltages appear frequently from about $8 \mathrm{kA}$ in a charging process, and these disappear while holding the current or discharging to about 9 $\mathrm{kA}$. These should be due to the conductor motions which have hysteresis. Positive and negative voltages are due to the motions in $\mathrm{H} 1$ and $\mathrm{H} 2$ coils, respectively. The amplitude of the spike voltage should be proportional to the current and not the velocity but the amount of the motion, because the signal was cut by a low-pass filter of $10 \mathrm{~Hz}$. Besides, the balance voltages with fixed shapes appear when changing the ramp rate of the current. These are considered to be induced by the secondary circuits such as the helical coil cases and the supporting structures.

In the first excitation tests of the third cooling period, the number and amplitude of the spike voltages were clearly larger than the excitations in the latter half of the second cooling period [7]. A normal-zone propagation and recover was observed when reaching $11.33 \mathrm{kA}$, as shown in Fig. 5 . Since the resistive voltage drop given by $(10)$ was positive, a normal zone propagated in the H1-I block. Slow excitation may not be effective for improving the cryogenic stability, and training effects by repeated excitations will be partially lost by warm-up.

TABLE I

Major Excitation TESTS in the 2 ND ANd 3Rd CoOlng PeRions

\begin{tabular}{|c|c|}
\hline Date & Current (mode_field@ major radius) \\
\hline Oct. 20 '98 & $10.41 \mathrm{kA}(\# 1-0.2 .50 \mathrm{~T} @ 3.75 \mathrm{~m})(0.1 \mathrm{~T} / \mathrm{min})$ \\
\hline Oct. 21 & $11.45 \mathrm{kA}\left(\# 1-0 \_2.75 \mathrm{~T} @ 3.75 \mathrm{~m}\right)$ wide propagation \\
\hline Dec. 1 & $9.16 \mathrm{kA}(\# 1-0.2 .20 \mathrm{~T} @ 3.75 \mathrm{~m})$ \\
\hline Dec. 3 & 10.62 kA (\#1-o_2.55 T@3.75 m) \\
\hline Dec. 10 & $11.25 \mathrm{kA}\left(\# 1-0 \_2.70 \mathrm{~T} @ 3.75 \mathrm{~m}\right)$ \\
\hline Dec, 16 & $11.25 \mathrm{kA}\left(\# 1-\mathrm{b} \_2.70 \mathrm{~T} @ 3.75 \mathrm{~m}\right), 10.41 \mathrm{kA}\left(\# 1-\mathrm{c} \_2.50 \mathrm{~T}\right)$ \\
\hline Dec. 17 & $\begin{array}{l}11.41 \mathrm{kA}\left(\# 1-0 \_2.74 \mathrm{~T} @ 3.75 \mathrm{~m}\right) \text {, } \\
11.40 \mathrm{kA}(\# 1-\mathrm{d} 2.85 \mathrm{~T} @ 3.60 \mathrm{~m})\end{array}$ \\
\hline Dec. 18 & $11.45 \mathrm{kA}\left(\# 1-0 \_2.75 \mathrm{~T} @ 3.75 \mathrm{~m}\right)$ \\
\hline July 7 '99 & $8.33 \mathrm{kA}\left(\# 1-0 \_2.00 \mathrm{~T} @ 3.75 \mathrm{~m}\right)$ \\
\hline July 8 & $10.41 \mathrm{kA}\left(\# 1-0 \_2.50 \mathrm{~T} @ 3.75 \mathrm{~m}\right)$ \\
\hline July 21 & $11.33 \mathrm{kA}\left(\# 1-0 \_2.72 \mathrm{~T} @ 3.75 \mathrm{~m}\right)\left({ }^{*} 1\right)$ \\
\hline July 22 & $11.20 \mathrm{kA}\left(\# 1-\mathrm{d} \_2.80 \mathrm{~T} @ 3.60 \mathrm{~m}\right)$ \\
\hline Aug. 6 & $11.40 \mathrm{kA}\left(\# 1-\mathrm{d} \_2.85 \mathrm{~T} @ 3.60 \mathrm{~m}\right)$ \\
\hline Aug. 25 & $11.40 \mathrm{kA}\left(\# 1-\mathrm{d} \_2.85 \mathrm{~T} @ 3.60 \mathrm{~m}\right)$ \\
\hline Sep. 9 & $11.27 \mathrm{kA}\left(\# 1-\mathrm{d} \_2.90 \mathrm{~T} @ 3.50 \mathrm{~m}\right)$ \\
\hline Nov. 30 & $\begin{array}{l}\text { av. } 11.65 \mathrm{kA}\left(\# 1-\mathrm{d} \_2.91 \mathrm{~T} @ 3.60 \mathrm{~m}\right)\left({ }^{*} 1\right) \\
(\mathrm{H}-\mathrm{I} / \mathrm{M} / \mathrm{O}=11.08 / 11.84 / 12.03 \mathrm{kA})\end{array}$ \\
\hline Dec. 15 & $\begin{array}{l}11.41 \mathrm{kA}(\# 1-\mathrm{o}-2.74 \mathrm{~T} @ 3.75 \mathrm{~m}) \\
\text { av. } 11.65 \mathrm{kA}\left(\# 1-\mathrm{d} \_2.91 \mathrm{~T} @ 3.60 \mathrm{~m}\right)\left({ }^{*} 1\right)\end{array}$ \\
\hline
\end{tabular}

(*1) A normal zone propagated and recovered. (a) Charge up to \#l-o_2.7 T (July 21, 1999)

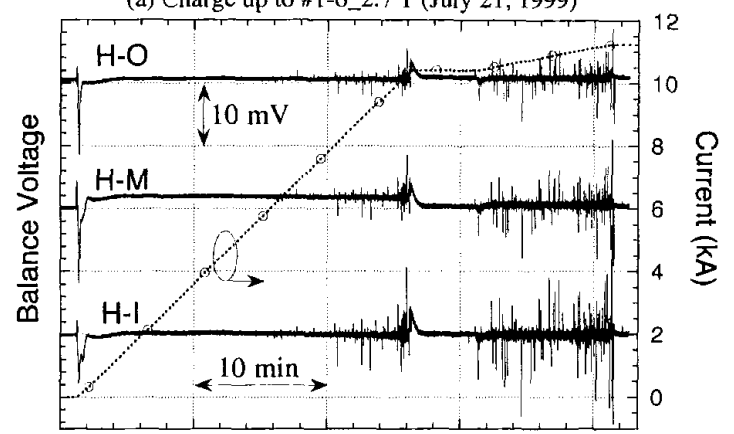

(b) Discharge from \#1-o_2.7 T (July 21, 1999)

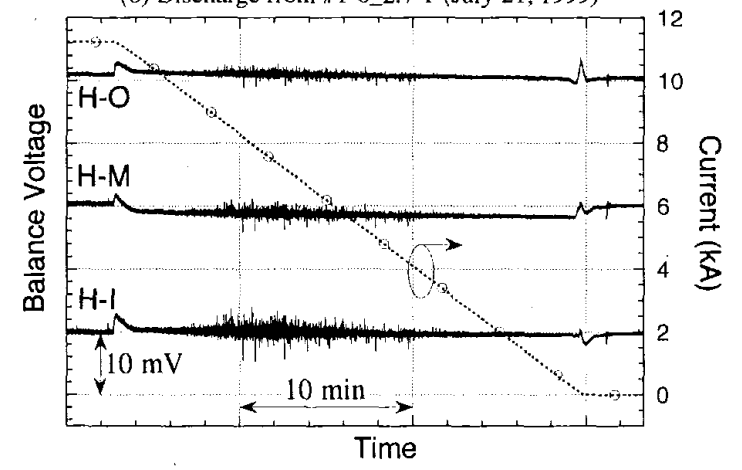

Fig. 4. Balance voltage of the helical coils during a charge and a discharge. The current was estimated from the magnetic field near the coil.
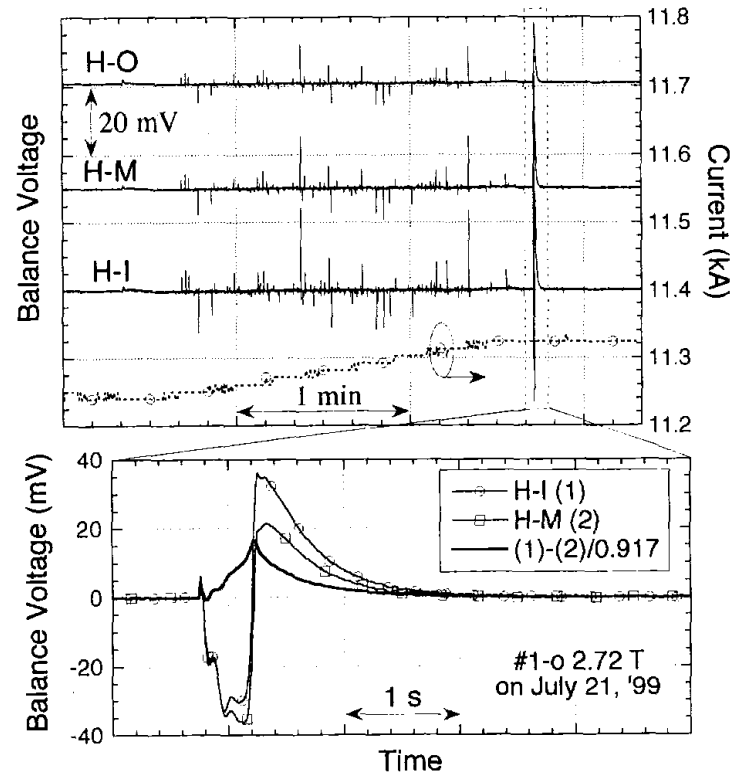

Fig, 5. Balance voltage of the helical coils when charging from \#1-0_2.70 T to $\# 1-\mathrm{o} \_2.72 \mathrm{~T}$ by $0.01 \mathrm{~T} / \mathrm{min}$ on July 21,1999 .

Since the maximum magnetic field in $\mathrm{H}-\mathrm{M}$ and $\mathrm{O}$ blocks is lower than H-I block [8], these should be charged up to the higher current within cryostable conditions. We have tried a current-grading excitation up to average $11.67 \mathrm{kA}$. A normalzone propagation and recover occurred in the H1-I block at average $11.65 \mathrm{kA}$, as shown in Fig. 6. The same excitation was tried to confirm the coil training effect. However, a 
normal zone was induced at almost the same current. A large disturbance able to induce a normal zone might occur at several positions in the coils.

\section{Discussion}

\section{A. Disturbance in the Helical Coils}

The spike voltages are coincidentally observed in the three blocks, and the clear voltages of H-I block are always higher than the other blocks. Therefore, apparent conductor motions are induced in only the H-I block. From the comparison of charging and discharging processes, the spike voltages in discharging are obviously lower and more frequent than those in charging. Consequently, the conductors move back smoothly in a discharging process. It is not clear why and where large motions are induced in only the H-I block in a charging process, but it should be related to the structure of the coils. Since total displacements of the H-M and O blocks by electromagnetic forces are less than the $\mathrm{H}-\mathrm{I}$ block, these blocks might be within an elastic region, or the motions might be smooth by being pressed by the H-I block.

\section{B. Cryogenic Stability of the Helical Coil}

The transitions of the length of the normal zones are shown in Fig. 6 that shows estimations by (10) and (11) are in good agreement. The normal zones recovered by a characteristic time constant, which may be caused by the resistance of current transfer. In the case of December 15, the length of a normal zone stagnated for about $2 \mathrm{~s}$. The normal zone might have started recovering at one side before another side.

There is a threshold current depending on the field, beyond which a normal zone can propagate dynamically by excess heat generation during the current diffusing into a pure aluminum stabilizer. When a current is slightly higher than the threshold, the normal zone can recover because of a ripple of the magnetic field or the current penetration in the initial normal zone. The threshold of the H-I block becomes clear as shown in Fig. 7, but that of the H-M or O block should be lower because of the larger spacer rate or the effect of the direction of the field. Further evaluation and basic experiments are necessary for the higher excitation.

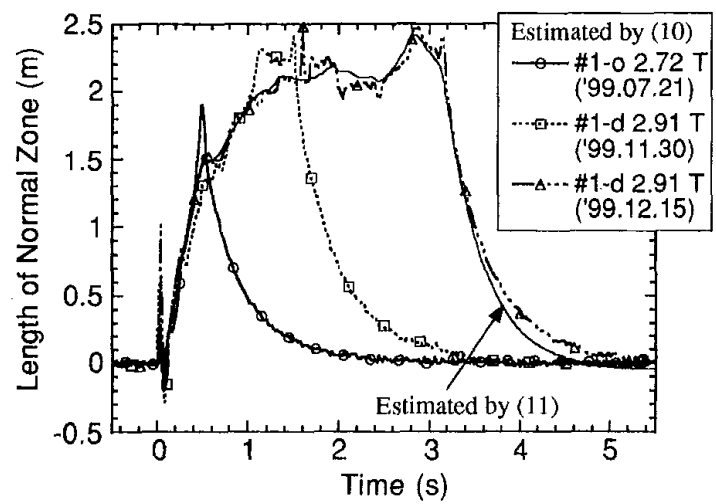

Fig. 6. A normal-zone propagation and recovery in the 3 rd cooling period. The length was estimated by using average resistance of $0.77 \mu \Omega / \mathrm{m}$.

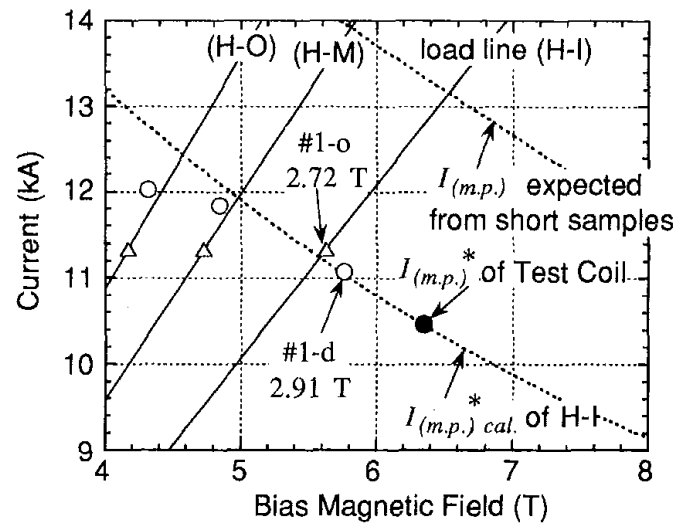

Fig. 7. A dynamic normal-zone propagating current $l(m . p .)^{*}$ and the coldend recovery current $I(m . p$.$) .$

\section{SUMMARY}

The helical coils of LHD are pool-cooled superconducting coils made of large composite conductors stabilized by a pure aluminum. A normal zone can propagate dynamically below the cold-end recovery current by excess heat generation during the current diffusion. Although disturbances due to the conductor motions seem to be reduced in the second and later excitations, this effect seems to be lost by warm-up. Large disturbances to initiate a normal zone will occur at the first higher excitation in every cooling period. The coils were successfully excited up to average $11.65 \mathrm{kA}$ by grading the current in the three blocks, and the disturbance does not occur in the beginning of a discharging process. The coils have been used safely for plasma experiments by being charged once up to the slightly higher current. Furthermore, a higher excitation is expected by current-grading of the larger ratio and by improving cooling condition.

\section{ACKNOWLEDGMENT}

The authors are indebted to the staffs of the LHD project as well as many collaborators from universities.

\section{REFERENCES}

[1] A. Ijyoshi, M. Fujiwara, O. Motojima, N. Ohyabu and K. Yamazaki, "Design study for the Large Helical Device", Fusion Technology, vol. 17, pp. 169-187, 1990 .

[2] N. Yanagi, T. Mito, S. Imagawa, et al., "Development and quality control of the superconductors for the helical coils of LHD", Fusion Eng. Des., vol. 41, pp. 241-246, 1998.

[3] S. Noguchi, A. Ishiyama, T. Satow and N. Yanagi, "Transient stability of large aluminum stabilized superconductors", IEEE Trans. Appl. Supercond., vol. 9, pp. 624-627, 1999.

[4] N. Yanagi, A. V. Gavrilin, T. Mito, et al., "Stability characteristics of the aluminum stabilized superconductor for the LHD helical coils", Advances in Superconductivity XI, pp. 991-994, 1999.

[5] S. Imagawa, N. Yanagi, H. Chikaraishi, et al., "Results of the first excitation of helical coils of the Large Helical Device", IEEE Trans. Appl. Supercond., Vol. 10, pp. 606-609, 2000.

[6] H. Chikaraishi, S. Yamada, O. Motojima, T. Sato, K. Tomatsu, H Niwa and T. Haga, "Current control of superconducting coils of LHD", Proc Fusion Technology 1998 , pp. 759-762, 1998.

[7] N. Yanagi, S. Imagawa, S. Hamaguchi, et al., "Pulse height analysis of the spike signals on the balance voltage observed in the LHD helical coils", Proc. ICEC 18, Bombay, 2000, submitted for publication.

[8] S. Imagawa, N. Yanagi, T. Satow, J. Yamamoto, O. Motojima and the LHD group, "Optimization of wetted surface fraction of helical coil for LHD”, Cryogenics, vol . 34, pp. 701-704, 1994. 